\title{
ANALYSIS OF LARGE DEFORMATION OF A HEAVY CANTILEVER*
}

\author{
SZE-BI HSU† AND SHIN-FENG HWANG $\ddagger$
}

\begin{abstract}
In this paper a mathematical model is discussed describing the deformation of a cantilever by its own weight. We assume that a cantilever of uniform cross-section and density is held fixed at an angle $\alpha$ at one end and is free at the other end. The shape of the cantilever depends heavily on $\alpha$ and a nondimensional parameter $K$ which represents the relative importance of density and length to that of flexural rigidity. We analyze the bifurcation phenomena for the vertical case, $\alpha=\pi$. Several numerical results are presented and discussed.
\end{abstract}

Key words. bifurcation, Sturm comparison, nonlinear eigenvalue problem, nonlinear oscillation

AMS(MOS) subject classifications. 73K05, 34B15, 34C10, 34C15

1. Introduction. The deformation of a cantilever by its own weight is of interest both practically due to its engineering significance and theoretically due to its inherent nonlinearity. We assume that a cantilever of uniform cross-section and density is held fixed at an angle $\alpha$ at one end and is free at the other end. If the cantilever is thin enough then its deformed shape can be described by the elastica theory. Using this approximation and small deflections, Euler first investigated the stability of a vertical cantilever (column) under its own weight [3]. Euler's stability problem was later corrected by Greenhill [4] who obtained the minimum unstable height for a column of given density and rigidity. The large deformation of a heavy elastica was first numerically integrated by Bickeley [1] who found only one of the solutions of the originally horizontal cantilever. Later, Wang [8] used the perturbation method on the elastica equations for a small and large parameter $K$, where $K$ is a nondimensional parameter which represents the relative importance of density and length to that of flexural rigidity. In [8] Wang also studied the bifurcation phenomena numerically as the parameters $K, \alpha$ change.

In this paper we first give the uniqueness results for the solutions of the elastica equation. We then give the complete bifurcation results for the vertical case, $\alpha=\pi$, in the spirit of [6], [7]. From these analytic results we improve the numerical results in [8] and give the reliable numerical computation results.

2. Formulation. We assume a cantilever of uniform density $\rho$ and total length $L$, is held fixed at an angle $\alpha$ at one end, say, the origin, and is free at the other end. Let us consider a small segment of the cantilever. A moment balance gives (see Fig. 1)

$$
m-\rho\left(L-s^{\prime}\right) \sin \theta d s^{\prime}=m+d m,
$$

where $m=m\left(s^{\prime}\right)$ is the local moment, $s^{\prime}$ is the arc length from the origin, and $\theta=\theta\left(s^{\prime}\right)$ is the local angle of inclination. According to Euler, the local moment is proportional to the curvature $d \theta / d s^{\prime}$, i.e.,

$$
m=-\mathrm{EI} \frac{d \theta}{d s^{\prime \prime}}
$$

\footnotetext{
* Received by the editors August 6, 1986; accepted for publication (in revised form) June 9, 1987.

$\dagger$ Institute of Applied Mathematics, Tsing-Hua University, Hsinchu, Taiwan, Republic of China. The research of this author was supported in part by National Research Council, Republic of China.

$\ddagger$ Department of Applied Mathematics, Chiao-Tung University, Hsinchu, Taiwan, Republic of China.
} 

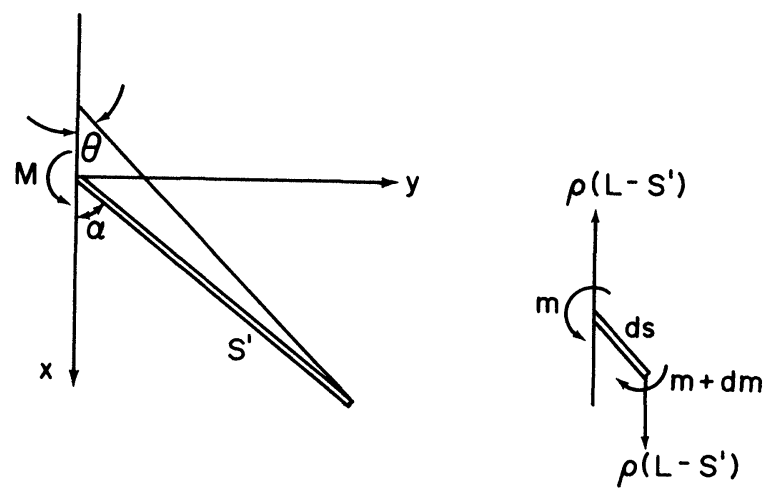

FIG. 1

where EI is the flexural rigidity of the material. From (2.1), (2.2), we obtain

$$
\mathrm{EI} \frac{d^{2} \theta}{d s^{\prime 2}}=\rho\left(L-s^{\prime}\right) \sin \theta
$$

and the boundary conditions are

$$
\theta(0)=\alpha, \quad \frac{d \theta}{d s^{\prime}}(L)=0 .
$$

Let $s=s^{\prime} / L$ and then (2.3), (2.4) become

$$
\begin{aligned}
& \frac{d^{2} \theta}{d s^{2}}=K^{3}(1-s) \sin \theta, \quad K>0, \quad 0 \leqq s \leqq 1, \\
& \theta(0)=\alpha, \quad \theta^{\prime}(1)=0, \quad-\pi<\alpha<\pi .
\end{aligned}
$$

The important parameter $K=\left(\rho L^{3} / \mathrm{EI}\right)^{1 / 3}$ represents the relative importance of density and length to that of flexural rigidity.

The main concern of this paper is to determine the multiplicities of solutions of (2.5) provided that $K>0,-\pi \leqq \alpha \leqq \pi$ are given.

First of all, we shall reformulate our problem (2.5). Let

$$
\psi(s)=\theta(1-s), \quad 0 \leqq s \leqq 1 .
$$

Then (2.5) becomes

$(\mathrm{P})_{\alpha}$

$$
\frac{d^{2} \psi}{d s^{2}}=K^{3} s \sin \psi, \quad 0<s<1, \quad K>0,
$$

$$
\psi^{\prime}(0)=0, \quad \psi(1)=\alpha, \quad-\pi \leqq \alpha \leqq \pi .
$$

Since $\psi(s), 0 \leqq s \leqq 1$, is a solution of $(\mathrm{P})_{\alpha}$ if and only if $-\psi(s)$ is a solution of $(\mathrm{P})_{-\alpha}$. Hence we only consider the problem with $0 \leqq \alpha \leqq \pi$. We may also reduce the problem (P) $\alpha, 0 \leqq \alpha \leqq \pi$, by the following scaling:

$$
\Psi(s)=\psi(s / K) .
$$

Then $\Psi(s)$ satisfies

$$
\begin{aligned}
& \frac{d^{2} \Psi}{d s^{2}}=s \sin \Psi(s), \quad 0 \leqq s \leqq K, \\
& \Psi^{\prime}(0)=0, \quad \Psi(K)=\alpha, \quad 0<\alpha<\pi .
\end{aligned}
$$


3. Uniqueness of solutions of $(P)_{\alpha}, 0 \leqq \alpha \leqq \pi$. In this section, we present some results concerning the uniqueness of solutions of boundary value problem

$(\mathrm{P})_{\alpha}$

$$
\frac{d^{2} \psi}{d s^{2}}=K^{3} s \sin \psi, \quad 0 \leqq s \leqq 1, \quad K>0,
$$

$$
\psi^{\prime}(0)=0, \quad \psi(1)=\alpha, \quad 0 \leqq \alpha \leqq \pi .
$$

LEMMA 3.1. The problem $(\mathrm{P})_{0}$ has a unique solution, namely,

$$
\psi(s) \equiv 0, \quad 0 \leqq s \leqq 1 \quad \text { for any } K>0 .
$$

Proof. Obviously $\psi(s) \equiv 0$ is a solution of $(\mathrm{P})_{0}$. Multiplying the equation in $(\mathrm{P})_{0}$ by $d \psi / d s$ and integrating the resulting equation from 0 to 1 , we obtain

$$
\frac{1}{2}\left(\psi^{\prime}(1)\right)^{2}=K^{3}\left[\int_{0}^{1} \cos \psi(s) d s-1\right] \geqq 0 .
$$

However,

$$
\int_{0}^{1} \cos \psi(s) d s-1 \leqq 0 .
$$

Hence we have $\psi^{\prime}(1)=0$. Since $\psi(1)=0, \psi^{\prime}(1)=0$, the conclusion $\psi(s) \equiv 0$ follows directly from the uniqueness of solutions of ordinary differential equations.

The existence of solution of problem $(\mathrm{P})_{\alpha}$ follows directly from the results in [2] since the right-hand side of $(\mathrm{P})_{\alpha}, K^{3} s \sin \psi$, is a bounded function for $0 \leqq s \leqq 1$.

We now present a result concerning the uniqueness of solution of $(\mathrm{P})_{\alpha}$.

LEMMA 3.2. If $K^{3}<\sqrt{45}$, then (P) $)_{\alpha}$ has a unique solution for every $\alpha \in[0, \pi]$.

Proof. Let $\psi(s)$ be a solution of $(\mathrm{P})_{\alpha}$; then

$$
\psi(s)=\alpha-\int_{0}^{1} K^{3} \xi \sin \psi(\xi) G(s, \xi) d \xi
$$

where

$$
G(s, \xi)=1-\max (s, \xi) .
$$

Let $\psi_{1}(s), \psi_{2}(s)$ be solutions of $(\mathrm{P})_{\alpha}$. Then

$$
\begin{aligned}
\left|\psi_{1}(s)-\psi_{2}(s)\right| & \leqq \int_{0}^{1} G(s, \xi) \xi\left|\psi_{1}(\xi)-\psi_{2}(\xi)\right| d \xi \\
& \leqq K^{3}\left[\int_{0}^{1} G^{2}(s, \xi) \xi^{2} d \xi\right]^{1 / 2}\left\|\psi_{1}-\psi_{2}\right\|_{2},
\end{aligned}
$$

or

$$
\begin{aligned}
\left\|\psi_{1}-\psi_{2}\right\|_{2}^{2} & =\int_{0}^{1}\left|\psi_{1}(s)-\psi_{2}(s)\right|^{2} d s \\
& \leqq K^{6}\left(\int_{0}^{1} \int_{0}^{1} G^{2}(s, \xi) \xi^{2} d \xi\right)\left\|\psi_{1}-\psi_{2}\right\|_{2}^{2}
\end{aligned}
$$

since

$$
\int_{0}^{1} \int_{0}^{1} G^{2}(s, \xi) \xi^{2} d \xi d s=\frac{1}{45} .
$$

If $K^{6} / 45<1$ or $K^{3}<\sqrt{45}$ then we must have

$$
\psi_{1} \equiv \psi_{2} \text {. }
$$


4. The multiplicities of the solutions of $(\mathrm{P})_{\alpha}$ for $\alpha=\pi$. In this section we shall present the analytic results for the vertical case, $\alpha=\pi$. The analytic results for this special case will help us to understand the bifurcation phenomena for the general problem $(\mathrm{P})_{\alpha}$. In the rest of this section, we shall restrict our attention to the vertical case, $\alpha=\pi$ :

$$
\frac{d^{2} \psi}{d s^{2}}=K^{3} s \sin \psi, \quad \psi^{\prime}(0)=0, \quad \psi(1)=\pi
$$

Let $s=x, v(x)=\psi(x / K)-\pi$. Then (4.1) takes the form

$$
\begin{aligned}
& v^{\prime \prime}(x)+x \sin v=0, \quad '=d / d x, \\
& v^{\prime}(0)=0, \quad v(K)=0 .
\end{aligned}
$$

We shall study the boundary value problem (4.2) by the shooting method and consider the following initial value problem

$$
\begin{aligned}
& v^{\prime \prime}(x)+x \sin v=0, \\
& v^{\prime}(0)=0, \\
& v(0)=a, \quad a \in \mathbb{R} .
\end{aligned}
$$

We denote the solution of (4.3) by $v(x, a)$. From the uniqueness of solutions of ordinary differential equations, it follows that

$$
\begin{aligned}
& v(x, 2 \pi+a)=2 \pi+v(x, a), \\
& v(x, 2 \pi-a)=2 \pi-v(x, a), \\
& v(x, a)=-v(x,-a), \\
& v(x, 0) \equiv 0, \quad v(x, \pi) \equiv \pi .
\end{aligned}
$$

From (4.4), we shall consider $v(x, a)$ only for $0<a<\pi$.

LEMMA 4.1. Let $0<a<\pi$. Then

(i) $-\pi / 2<v(x, a)<\pi / 2$ for $0<a<\pi / 2, x \geqq 0$.

(ii) $-\pi<v(x, a)<\pi$ for $\pi / 2 \leqq a<\pi, x \geqq 0$.

(iii) $v(x, a)$ is oscillatory over $[0, \infty)$ for all $0<a<\pi$.

Proof. Multiplying (4.3) by $v^{\prime}(x)$ and integrating the resulting equation from 0 to $x$, we obtain

$$
\frac{1}{2}\left(v^{\prime}(x)\right)^{2}=x \cos v(x)-\int_{0}^{x} \cos v(\xi) d \xi \geqq 0 .
$$

If $0<a<\pi / 2$, then $\cos a=\cos v(0)>0$. We claim that $\cos v(x)>0$ for all $x \geqq 0$. If not, then there exists $x_{0}>0$ such that $\cos v(x)>0$ for all $0 \leqq x<x_{0}$ and $\cos v\left(x_{0}\right)=0$. Then this contradicts (4.5) with $x=x_{0}$ and we complete the proof for (i).

If $\pi / 2 \leqq a<\pi$, then $\cos a=\cos v(0) \in(-1,0]$. We claim that $\cos v(x) \neq-1$ for all $x \geqq 0$. If not, then there exists $x_{0}>0$ such that $\cos v\left(x_{0}\right)=-1$ and $\cos v(x)>1$ for $0 \leqq x<x_{0}$. Again from (4.5) we obtain a contradiction. Hence $-\pi<v(x, a)<\pi$ for all $x \geqq 0$ and we established (ii).

We next show that $v(x, a)$ is oscillatory over $[0, \infty)$ for any $0<a<\pi$. Let

$$
V(x)=(1-\cos v(x))+\frac{1}{2} \frac{\left(v^{\prime}(x)\right)^{2}}{x} .
$$


It is easy to verify that

$$
V^{\prime}(x)=-\frac{1}{2}\left(\frac{v^{\prime}(x)}{x}\right)^{2} \leqq 0
$$

Then we have

$$
1-\cos v(x) \leqq V(x) \leqq V(0)=1-\cos a .
$$

Since $-\pi<v(x)<\pi$, we then have $|v(x)| \leqq a$ for all $x \geqq 0$. We rewrite the equation in (4.3) as

$$
v^{\prime \prime}(x)+x\left(\frac{\sin v(x)}{v(x)}\right) v(x)=0 .
$$

Let $0<\delta<\min _{0 \leqq v \leqq a}(\sin v / v)$. Using Sturm's comparison theorem [5], we compare (4.6) with

$$
v^{\prime \prime}+\delta v=0
$$

which is oscillatory over $[0, \infty)$. Thus we complete the proof for (iii).

Next we introduce the following notation:

$$
\Delta(x, a)=\frac{d v}{d a}(x, a), \quad \phi(x)=\Delta(x, 0) .
$$

Differentiating (4.3) with respect to $a$ yields

$$
\Delta^{\prime \prime}(x)+x(\cos v(x, a)) \Delta(x)=0, \quad \Delta(0)=1, \quad \Delta^{\prime}(0)=0 .
$$

Setting $a=0$ in (4.8) yields

$$
\phi^{\prime \prime}(x)+x \phi(x)=0, \quad \phi(0)=1, \quad \phi^{\prime}(0)=0 .
$$

The equation in (4.9) is the well-known Airy equation which is oscillatory over $[0, \infty)$. Let $\lambda_{n}, \gamma_{n}$ be the $n$th zero of $\phi(x)$ and $\phi^{\prime}(x)$, respectively, for $n=1,2, \cdots$. We note that

$$
\begin{aligned}
& \lambda_{1} \approx 1.98635, \quad \lambda_{2} \approx 3.82557, \quad \lambda_{3} \approx 5.29566, \\
& \lambda_{4} \approx 6.58432, \text { etc. (See Fig. } 2 \text {.) }
\end{aligned}
$$

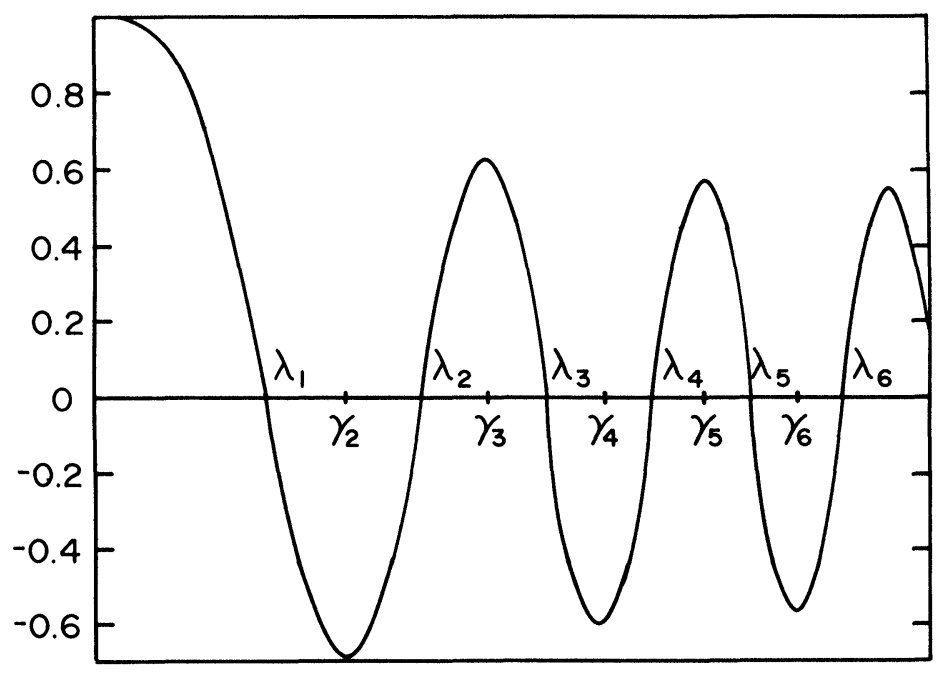

FIG. 2 
From Lemma 4.1(iii), $v(x, a)$ is oscillatory over [0, $\infty)$ for any $0<a<\pi$. Let $y_{n}(a)$, $z_{n}(a)$ be the $n$th zero of $v(x, a)$ and $v^{\prime}(x, a)$, respectively, for $n=1,2, \cdots, 0<a<\pi$. (See Fig. 3.)

LEMMA 4.2.

(i) $\lim _{a \rightarrow 0^{+}} y_{n}(a)=\lambda_{n}, \lim _{a \rightarrow 0^{+}} z_{n}(a)=\gamma_{n}$ for $n=1,2, \cdots$,

(ii) $\lim _{a \rightarrow \pi^{-}} y_{n}(a)=+\infty$ for $n=1,2, \cdots$.

Proof. The proof of (i) follows directly from the following identities [6]:

$$
\begin{aligned}
\lim _{a \rightarrow 0^{+}} \frac{v(x, a)}{a} & =\lim _{a \rightarrow 0^{+}} \frac{v(x, a)-v(x, 0)}{a} \\
& =\lim _{a \rightarrow 0^{+}} \frac{d v}{d a}\left(x, \delta_{a}\right), \quad 0<\delta_{a}<a \\
& =\frac{d v}{d a}(x, 0)=\phi(x),
\end{aligned}
$$

and

$$
\begin{aligned}
\lim _{a \rightarrow 0^{+}} \frac{v^{\prime}(x, a)}{a} & =\lim _{a \rightarrow 0^{+}} \frac{v^{\prime}(x, a)-v^{\prime}(x, 0)}{a} \\
& =\lim _{a \rightarrow 0^{+}} \frac{d}{d a}\left(v^{\prime}\left(x, \delta_{a}\right)\right) \quad 0<\delta_{a}<a \\
& =\frac{d}{d x}\left(\frac{d}{d a} v(x, 0)\right)=\phi^{\prime}(x)
\end{aligned}
$$

since $v(x, \pi) \equiv \pi$ and $v(x, a)$ are oscillatory over $[0, \infty)$ for $0<a<\pi$. From continuous dependence on initial values, we obtain $\lim _{a \rightarrow \pi^{-}} y_{1}(a)=+\infty$ and hence $\lim _{a \rightarrow \pi^{-}} y_{n}(a)=$ $+\infty$ for $n=1,2, \cdots$. Thus we complete the proof for (ii).

In addition to the properties (i), (ii) in Lemma 4.2., we shall show that $y_{n}(a)$ satisfies

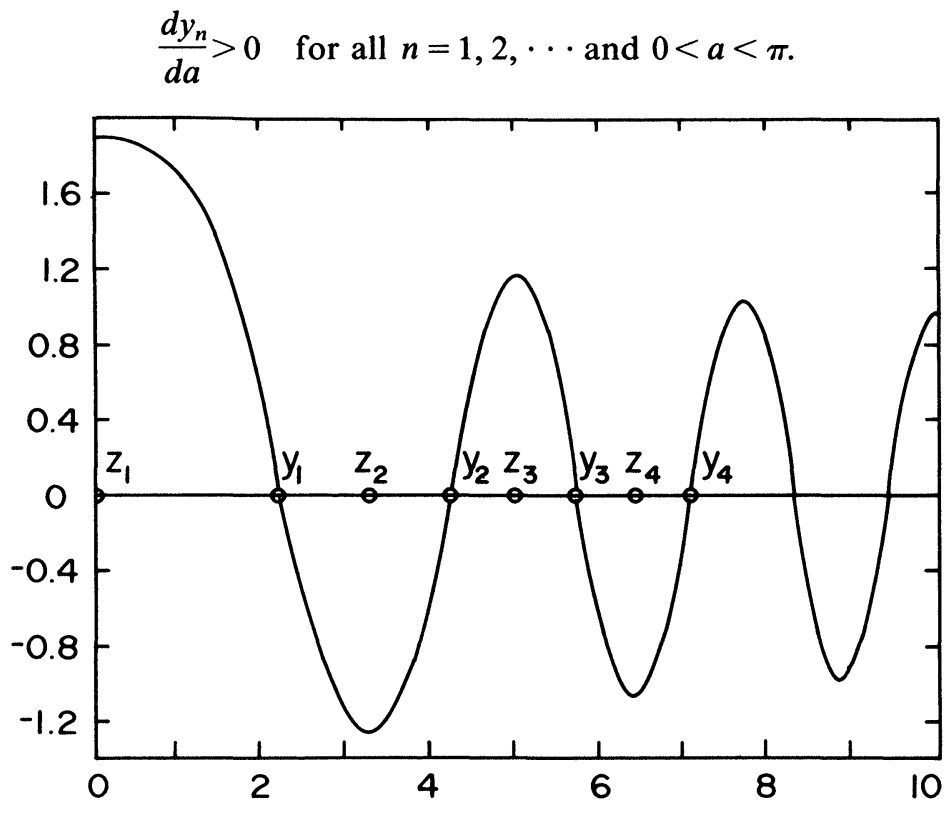

FIG. 3 
Assume that (4.11) holds; then we may plot the following graphs for $y_{n}(a), n=1,2, \cdots$. (See Fig. 4.) Then we conclude from (4.2) and (4.4) that

If $0<K<\lambda_{1}$ then (4.2) has the unique solution $v(x) \equiv 0$.

If $\lambda_{1}<K<\lambda_{2}$ then (4.2) has three distinct solutions.

If $\lambda_{n}<K<\lambda_{n+1}$ then (4.2) has $2 n+1$ distinct solutions.

Since

$$
v\left(y_{n}(a), a\right)=0, \quad 0<a<\pi,
$$

differentiating (4.12) with respect to $a$ yields

$$
v^{\prime}\left(y_{n}(a), a\right) \frac{d y_{n}}{d a}+\frac{d v}{d a}\left(y_{n}(a), a\right)=0
$$

or

$$
\frac{d y_{n}}{d a}=-\frac{\Delta\left(y_{n}(a), a\right)}{y^{\prime}\left(y_{n}(a), a\right)} .
$$

We now state our main result.

THEOREM 1. Let $0<a<\pi$.

(i) The solution $v(x, a)$ of (4.3) has an infinite number of isolated zeros $y_{n}(a)$, $y_{1}<y_{2}<\cdots<y_{n}$ and $y_{n} \rightarrow \infty$ as $n \rightarrow \infty$; likewise $v^{\prime}(x, a)$ has an infinite number of isolated zeros, $z_{n}(a), 0=z_{1}<z_{2}<\cdots<z_{n}$, interlacing the $y_{n}$; furthermore

$$
\lim _{a \rightarrow 0^{+}} y_{n}(a)=\lambda_{n}, \quad \lim _{a \rightarrow 0^{+}} z_{n}(a)=\gamma_{n},
$$

and

$$
\lim _{a \rightarrow \pi^{-}} y_{n}(a)=\infty \quad \text { for } n=1,2, \cdots .
$$

(ii) $y_{n}(a)$ is a differentiable function of $a$ and

$$
\frac{d y_{n}}{d a}>0 \quad \text { for } n=1,2, \cdots \text {. }
$$

We have shown part (i) in the above lemmas. The proof of (ii) follows directly from (4.13) and Lemma 4.3 below.

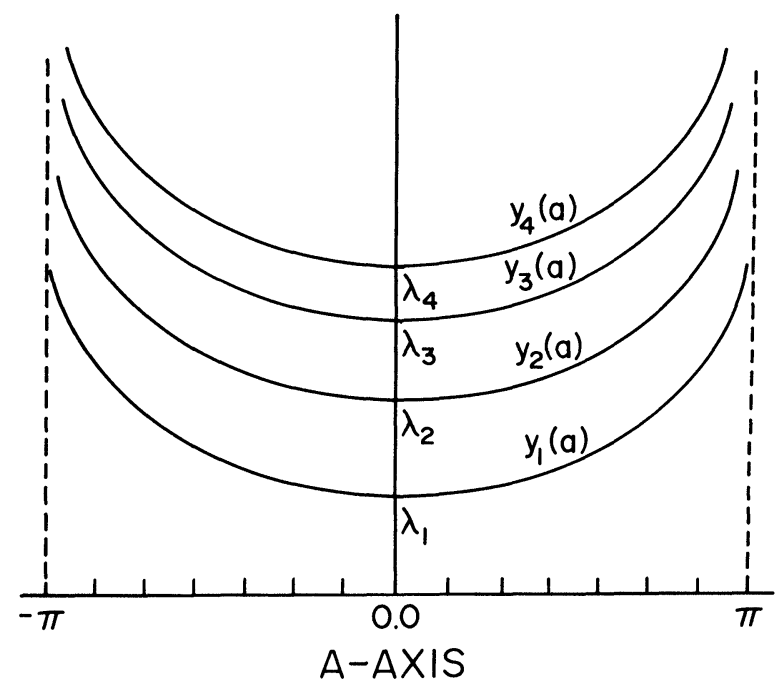

FIG. 4 
Lemma 4.3. Let $0<a<\pi$. Then $\Delta(x, a)$ has an infinite number of isolated zeros $\alpha_{n}(a), 0<\alpha_{1}<\cdots<\alpha_{n} . \Delta^{\prime}(x, a)$ satisfies the following:

(i) If $0<a<\pi / 2$, then $\Delta^{\prime}(x, a)$ has an infinite number of isolated zeros $\beta_{n}(a)$, $0=\beta_{1}<\beta_{2}<\cdots<\beta_{n}$. Furthermore $\beta_{1}=z_{1}=0<y_{1}<\alpha_{1}<z_{2}<\beta_{2}<y_{2}<\alpha_{2}<\cdots<y_{n}<$ $\alpha_{n}<z_{n+1}<\beta_{n+1}<y_{n+1}$. (See Fig. 5.)

(ii) If $\pi / 2 \leqq a<\pi$ then $\Delta^{\prime}(x, a)$ has an infinite number of isolated zeros $\beta_{n}(a)$, $0=\beta_{0}<\beta_{1}<\cdots<\beta_{n}$. Furthermore $\beta_{0}=z_{1}=0<\beta_{1}<y_{1}<\alpha_{1}<z_{2}<\beta_{2}<y_{2}<\cdots<y_{n}<$ $\alpha_{n}<z_{n+1}<\beta_{n+1}<y_{n+1}$. (See Fig. 6.)

Before we prove Lemma 4.3 we consider (4.3) and (4.8). Let

$$
\begin{aligned}
& v^{\prime \prime}+x \sin v=0, \quad v(0)=a, \quad v^{\prime}(0)=0, \\
& \Delta^{\prime \prime}+x(\cos v) \Delta=0, \quad \Delta(0)=1, \quad \Delta^{\prime}(0)=0 .
\end{aligned}
$$

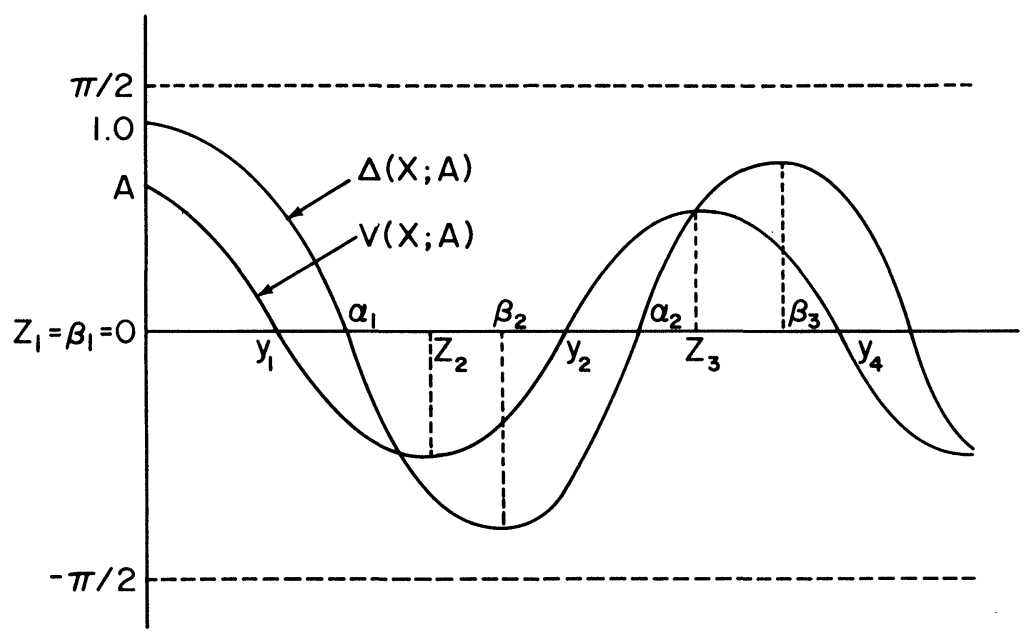

FIG. 5

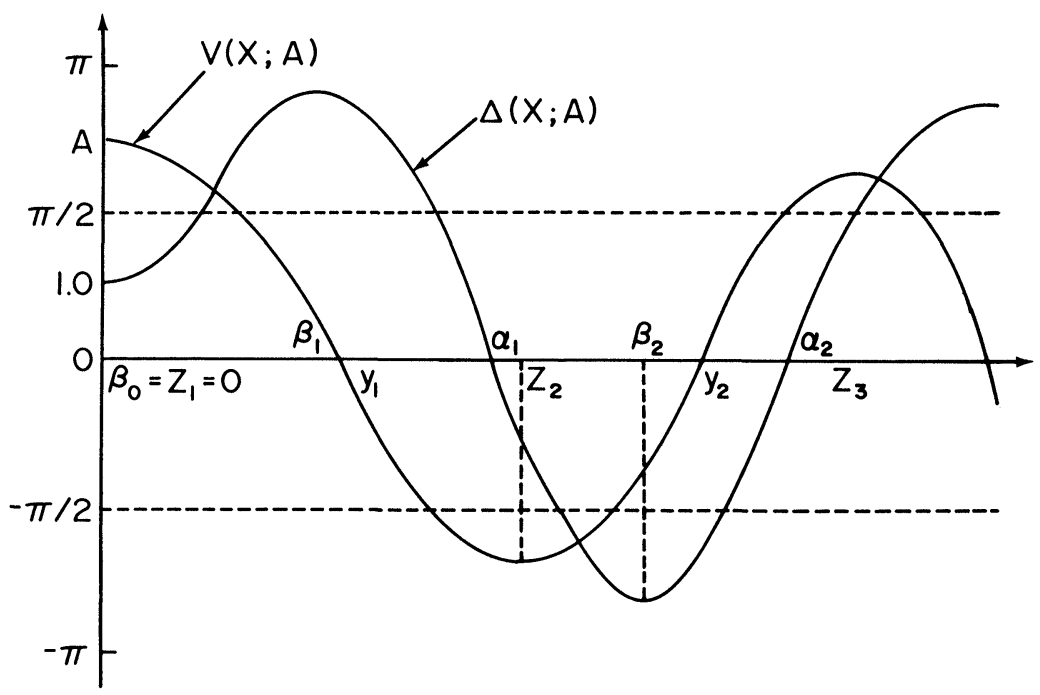

FIG. 6 
In addition to (A) and (B), we form the following equations satisfied by $\Delta^{\prime}$ and $\hat{v}=\left(x-3 y_{n}\right) v^{\prime}$, respectively:

$$
\begin{array}{ll}
\text { (C) } & \left(\Delta^{\prime}\right)^{\prime \prime}+x \cos v \Delta^{\prime}=\Delta\left(x v^{\prime} \sin v-\cos v\right), \\
\text { (D) } & \hat{v}^{\prime \prime}+x \cos v \hat{v}=-3\left(x-y_{n}\right) \sin v .
\end{array}
$$

Multiplying (A) by $\Delta$ and multiplying (B) by $v$, subtracting the resulting equations from each other and integrating the final expression from $\alpha$ to $\beta$, we obtain

$$
\left.\left(v^{\prime} \Delta-v \Delta^{\prime}\right)\right|_{\alpha} ^{\beta}=\int_{\alpha}^{\beta} x \Delta v\left(\cos v-\frac{\sin v}{v}\right) d x .
$$

Multiplying (A) by $\Delta^{\prime}$ and multiplying (C) by $v$, subtracting the resulting equations from each other and integrating the final expression from $\alpha$ to $\beta$, we obtain

(b) $\left.\left(v^{\prime} \Delta^{\prime}-v \Delta^{\prime \prime}\right)\right|_{\alpha} ^{\beta}=\int_{\alpha}^{\beta}\left\{-x \Delta^{\prime} \sin v+x \Delta^{\prime} v \cos v+\Delta v\left(\cos v-x v^{\prime} \sin v\right)\right\} d x$.

Multiplying (D) by $\Delta$ and multiplying (B) by $\hat{v}$, subtracting the resulting equation from each other and integrating the final expression from $\alpha$ to $\beta$, we obtain

$$
\left.\left(\hat{v}^{\prime} \Delta-\hat{v} \Delta^{\prime}\right)\right|_{\beta} ^{\alpha}=-\int_{\beta}^{\alpha} 3\left(x-y_{n}\right) \Delta \sin v d x .
$$

Finally we observe that, since $v^{\prime}(0)=0, v(0)=a, \Delta(0)=1, \Delta^{\prime}(0)=0,0<a<\pi$,

$$
\begin{array}{ll}
\operatorname{sg} v=(-1)^{n} & \text { for } y_{n}<x<y_{n+1}, \\
\operatorname{sg} v^{\prime}=(-1)^{n} & \text { for } z_{n}<x<z_{n+1}, \\
\operatorname{sg} \Delta=(-1)^{n} & \text { for } \alpha_{n}<x<\alpha_{n+1}, \\
\operatorname{sg} \Delta^{\prime}=(-1)^{n} & \text { for } \beta_{n}<x<\beta_{n+1} .
\end{array}
$$

Proof of Lemma 4.3. We shall prove the lemma by induction. We assume the truth of the statement up to $\alpha_{m}$, for $m=1$.

If $\pi / 2 \leqq a<\pi$, then we claim that $\beta_{1}<y_{1}$. If not, $\beta_{1} \geqq y_{1}$, then $\Delta^{\prime}(x)>0$ for all $0 \leqq x \leqq y_{1}$. We specialize $(\alpha, \beta)$ in $(\mathrm{b})$ to $\left(0, y_{1}\right)$. Then we obtain

$$
\begin{aligned}
v^{\prime} \Delta^{\prime}-\left.v \Delta^{\prime \prime}\right|_{0} ^{y_{1}}= & \int_{0}^{y_{1}}\left(v \Delta^{\prime} x \cos v-x \Delta^{\prime} \sin v+\Delta v \cos v\right) d x \\
& +\int_{0}^{y_{1}} x \Delta v\left(-v^{\prime} \sin v\right) d x
\end{aligned}
$$

Since

$$
\int_{0}^{y_{1}} x \Delta v\left(-v^{\prime} \sin v\right) d x=\left.x \Delta v \cos v\right|_{0^{1}} ^{y_{1}}-\int_{0}^{y_{1}} \cos v\left(x \Delta^{\prime} v+x \Delta v^{\prime}+\Delta v\right) d x
$$

and $v^{\prime}(0)=0, \Delta^{\prime \prime}(0)=0, v\left(y_{1}\right)=0$. Then (4.14) becomes

$$
\begin{aligned}
v^{\prime}\left(y_{1}\right) \Delta^{\prime}\left(y_{1}\right) & =\int_{0}^{y_{1}}-x \Delta^{\prime} \sin v d x-\int_{0}^{y_{1}} x \Delta v^{\prime} \cos v d x \\
& =\int_{0}^{y_{1}} \Delta \sin v d x .
\end{aligned}
$$

This is a desired contradiction for $v^{\prime}\left(y_{1}\right)<0, \Delta^{\prime}\left(y_{1}\right)>0$ and $\Delta(x)>0, \sin v(x)>0$ for $0 \leqq x<y_{1}$. 
We shall now show that $\alpha_{1}>y_{1}$ for $0<a<\pi$. If not, then there exists $\alpha^{*} \in\left(0, y_{1}\right)$ such that $\Delta\left(\alpha^{*}\right)=0, \Delta^{\prime}\left(\alpha^{*}\right)<0$ and $\Delta(x)>0$ for $0 \leqq x<\alpha^{*}$. We specialize $(\alpha, \beta)$ in (a) to $\left(0, \alpha^{*}\right)$. Then we have

$$
-v\left(\alpha^{*}\right) \Delta^{\prime}\left(\alpha^{*}\right)=\int_{0}^{\alpha^{*}} x \Delta v\left(\cos v-\frac{\sin v}{v}\right) d x .
$$

Since $\cos v \leqq \sin v / v$ for $-\pi<v<\pi$ and $\Delta(x)>0, v(x)>0$ for $0 \leqq x<\alpha^{*}$, it follows that the right-hand side of (4.16) is negative. However, the left-hand side of (4.16) is positive. This leads to a contradiction.

We now want to complete the induction. For $0<a<\pi$, we want to show the following:

(i) $y_{m}<\alpha_{m}<z_{m+1}$. By induction hypothesis $y_{m}<\alpha_{m}$. We want to show that $\alpha_{m}<z_{m+1}$. For $a=0$, it is obvious that $\alpha_{m}(0)=\lambda_{m}$. From Lemma 4.2, we have

$$
\lim _{a \rightarrow 0^{+}} z_{m+1}(a)=\gamma_{m+1}>\lambda_{m} .
$$

By continuous dependence on parameter $a$, we have that $\alpha_{m}(a)<z_{m+1}(a)$ for $a>0$ sufficiently small. We claim that $\alpha_{m}(a)<z_{m+1}(a)$ for all $0<a<\pi$. If not, there exists $a^{*} \in(0, \pi)$ such that $\alpha_{m}\left(a^{*}\right)=z_{m+1}\left(a^{*}\right)$. We now specialize $(\alpha, \beta)$ in (c) to $\left(z_{m}\left(a^{*}\right), z_{m+1}\left(a^{*}\right)\right)$ and $n=m$. Then we obtain

$$
\hat{v}^{\prime} \Delta-\left.\hat{v} \Delta^{\prime}\right|_{z_{m}} ^{z_{m+1}}=\int_{z_{m}}^{z_{m+1}} 3\left(x-y_{m}\right)(-\sin v(x)) \Delta(x) d x
$$

since $\hat{v}\left(z_{m+1}\right)=\hat{v}\left(z_{m}\right)=0, z_{m+1}=\alpha_{m}, \hat{v}^{\prime}\left(z_{m}\right)=\left(z_{m}-3 y_{m}\right) v^{\prime \prime}\left(z_{m}\right)$. Then (4.17) becomes

$$
0=\left(z_{m}-3 y_{m}\right) v^{\prime \prime}\left(z_{m}\right) \Delta\left(z_{m}\right)+\int_{z_{m}}^{z_{m+1}} 3\left(x-y_{m}\right)(-\sin v(x)) \Delta(x) d x .
$$

It is easy to verify that the right-hand side of (4.18) is positive. Then this is a desired contradiction. Hence, we have $\alpha_{m}(a)<z_{m+1}(a)$ for all $0<a<\pi$.

(ii) $z_{m+1}<\beta_{m+1}<y_{m+1}<\alpha_{m+1}$. First we show that $z_{m+1}<\beta_{m+1}$. If not, then $\alpha_{m}<$ $\beta_{m+1} \leqq z_{m+1}$. We specialize $(\alpha, \beta)$ in (a) to $\left(\alpha_{m}, \beta_{m+1}\right)$. Then we obtain

$$
v^{\prime}\left(\beta_{m+1}\right) \Delta\left(\beta_{m+1}\right)+v\left(\alpha_{m}\right) \Delta^{\prime}\left(\alpha_{m}\right)=\int_{\alpha_{m}}^{\beta_{m+1}} x \Delta v\left(\cos v-\frac{\sin v}{v}\right) d x .
$$

It is easy to verify that the left-hand side of (4.19) is positive while the right-hand side is negative. This is a contradiction.

Next we show that $\beta_{m+1}<y_{m+1}$. If not, then $\beta_{m+1} \geqq y_{m+1}$. We specialize $(\alpha, \beta)$ in

(b) to $\left(z_{m+1}, y_{m+1}\right)$. Following similar arguments in the case $\beta_{1}<y_{1}$, we deduce that

$$
v^{\prime}\left(y_{m+1}\right) \Delta^{\prime}\left(y_{m+1}\right)=z_{m+1} \Delta\left(z_{m+1}\right) \sin v\left(z_{m+1}\right)+\int_{z_{m+1}}^{y_{m+1}} \Delta \sin v d x .
$$

It is easy to verify $v^{\prime}\left(y_{m+1}\right) \Delta^{\prime}\left(y_{m+1}\right) \leqq 0, \quad z_{m+1} \Delta\left(z_{m+1}\right) \sin v\left(z_{m+1}\right)>0, \quad$ and $\int_{z_{m+1}}^{y_{m+1}} \Delta \sin v d x>0$. Thus we obtain a contradiction.

Finally, we want to show that $y_{m+1}<\alpha_{m+1}$. If not, then $y_{m+1} \geqq \alpha_{m+1}$. We specialize $(\alpha, \beta)$ in (a) to $\left(\beta_{m+1}, \alpha_{m+1}\right)$. Then we have

$$
-\left[v^{\prime}\left(\beta_{m+1}\right) \Delta\left(\beta_{m+1}\right)+v\left(\alpha_{m+1}\right) \Delta^{\prime}\left(\alpha_{m+1}\right)\right]=\int_{\beta_{m+1}}^{\alpha_{m+1}} x \Delta v\left(\cos v-\frac{\sin v}{v}\right) d x .
$$

It is easy to verify that the left-hand side of (4.20) is positive while the right-hand side is negative. This is a contradiction. 
5. Numerical studies for $\alpha \neq \pi$ and discussions. In this section, we present our numerical studies for the multiplicities of the solutions of the problem $(\mathrm{P})_{\alpha}, 0<\alpha<\pi$. The analytic results in $\S 4$ shall confirm that our numerical results are reliable.

Consider our bifurcation problem

$(\mathrm{P})_{\alpha}$

$$
\begin{aligned}
& \frac{d^{2} \psi}{d s^{2}}=K^{2} s \sin \psi, \quad K>0, \\
& \psi^{\prime}(0)=0, \quad \psi(1)=\alpha, \quad 0<\alpha<\pi
\end{aligned}
$$

and its scaled form

$$
\frac{d^{2} \Psi}{d s^{2}}=s \sin \Psi, \quad \Psi^{\prime}(0)=0, \quad \Psi(K)=\alpha .
$$

Let $\Psi(s, a)$ be the solution of the following initial value problem:

$$
\frac{d^{2} \Psi}{d s^{2}}=s \sin \Psi, \quad \Psi^{\prime}(0)=0, \quad \Psi(0)=a
$$

It is easy to verify the following relations:

$$
\begin{aligned}
& \Psi(s, a+2 \pi)=\Psi(s, a)+2 \pi, \\
& \Psi(s, 2 \pi-a)=2 \pi-\Psi(s, a) .
\end{aligned}
$$

For any $K>0$, we consider the map

$$
a \rightarrow \Psi(K, a), \quad 0 \leqq a \leqq 2 \pi .
$$

Since $0<\alpha<\pi$, from (5.3) we only need to compute numerically for $0<a<\pi$. In the following, we used the ODE Solver DGEAR of the IMSL Library to compute the function $\alpha=\Psi(K, \alpha), 0<a<\pi$, for various $K$.

In Fig. 7, the parameter $K$ satisfies $0<K=1.0<\lambda_{1} \approx 1.98635$ and the graph $\alpha=\Psi$ $(K, a)$ intersects $\alpha=\pi$ at only one point. We conjecture that for $0<K<\lambda_{1}$ the problem $(\mathrm{P})_{\alpha}$ has a unique solution for every $\alpha \in(0, \pi)$. In Fig. 8, the parameter $K$ satisfies

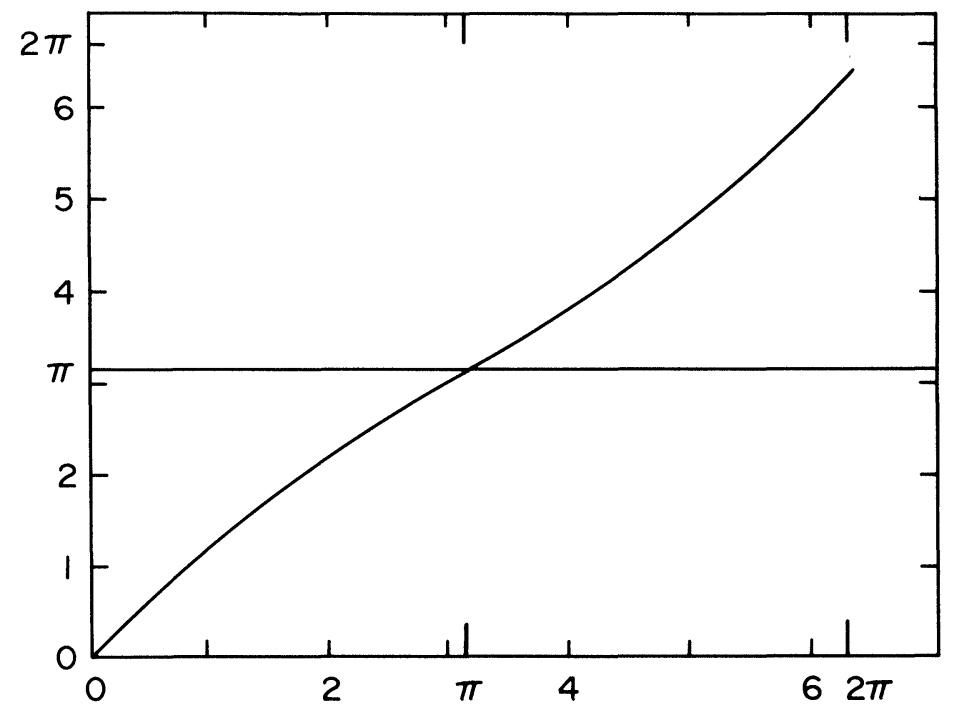

FIG. 7 


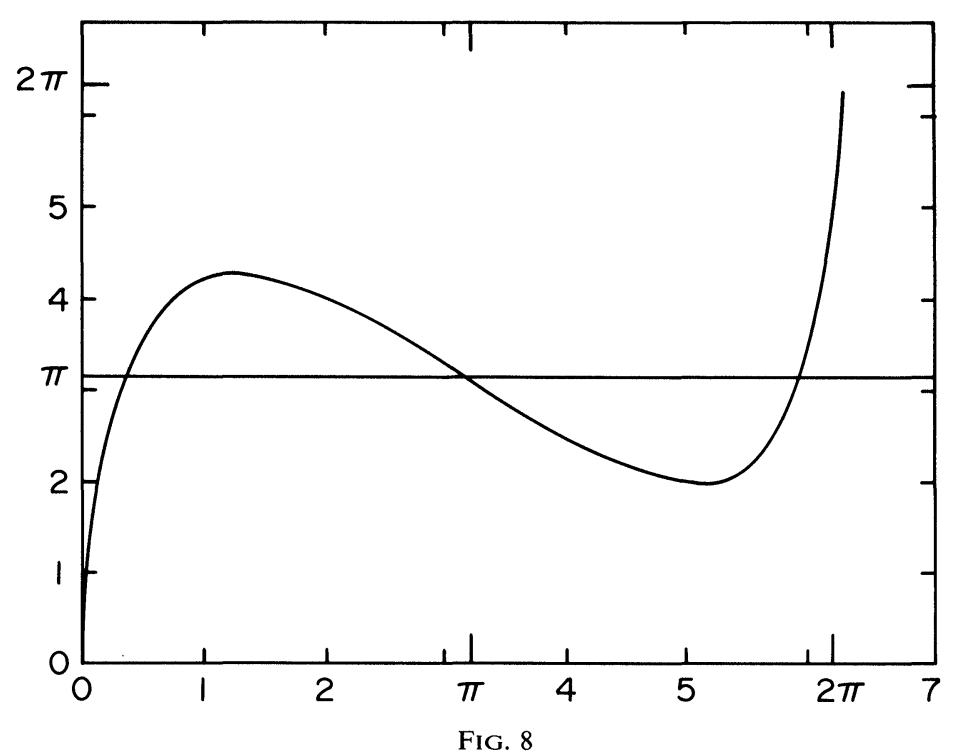

$\lambda_{1}<K=3.0<\lambda_{2} \approx 3.82557$ and the graph $\alpha=\Psi(K, a)$ intersects $\alpha=\pi$ at three distinct points. It shows that if $\lambda_{1}<K<\lambda_{2}$, then the problem $(\mathrm{P})_{\alpha}$ has at most three distinct solutions. In Fig. 9, the parameter $K$ satisfies $\lambda_{2}<K=4.5<\lambda_{3} \approx 5.29566$ and the graph $\alpha=\Psi(K, a)$ intersects $\alpha=\pi$ at five distinct points. It shows that if $\lambda_{2}<K<\lambda_{3}$, then the problem $(\mathrm{P})_{\alpha}$ has at most five distinct solutions. We conjecture that for $\lambda_{n}<K<\lambda_{n+1}$ the problem $(\mathrm{P})_{\alpha}$ has $1,3, \cdots, 2 n+1$ solutions for various $\alpha$.

Acknowledgments. We thank Professor C. Y. Wang of Michigan State University for suggesting this problem to us and for stimulating discussions. The authors also thank a referee for his comments.

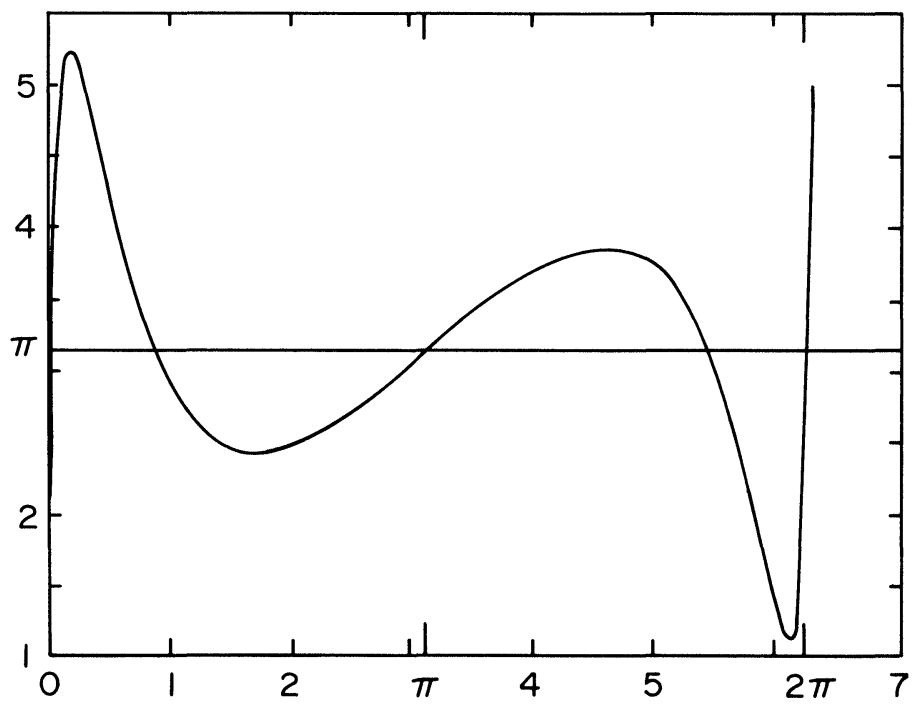

FIG. 9 


\section{REFERENCES}

[1] W. G. Bickeley, The heavy elastica, Phil. Mag. Ser., 717 (1934), pp. 603-622.

[2] S. N. ChOw, J. MAllet-PARET, AND J. YORKE, Finding zeros of maps: homotopy methods that are constructive with probability one, Math. Comp., 32 (1978), pp. 887-899.

[3] L. Euler, De curvis elasticis, 1744.

[4] A. G. Greenhill, Determination of the greatest height consistent with stability that a vertical pole or mast can be made, and of the greatest height to which a tree of given proportions can grow, Proc. Cambridge Philos. Soc., 4 (1881), pp. 66-78.

[5] P. Hartman, Ordinary Differential Equations, John Wiley, New York, 1964.

[6] I. I. Kolodner, Heavy rotating string-A nonlinear eigenvalue problem, Comm. Pure Appl. Math., 8 (1955), pp. 395-408.

[7] W. M. Ni, Uniqueness of solutions of nonlinear Dirichlet problems, J. Differential Equations, 50 (1983), pp. 289-304.

[8] C. Y. WANG, Large deformation of a heavy cantilever, Quart. Appl. Math., 39 (1981), pp. 261-273. 